\title{
Correction of Dispersion and the Betatron Functions in the CEBAF Accelerator*
}

\author{
V. A. Lebedev, M. Bickley, S. Schaffner, J. Van Zeijts \\ G. A. Krafft, C. Watson \\ Continuous Electron Beam Accelerator Facility, \\ 12000 Jefferson Ave., Newport News, VA 23606 USA
}

Abstract: During the commissioning of the CEBAF accelerator, correction of dispersion and momentum compaction, and, to a lesser extent, transverse transfer matrices were essential for robust operation. With changing machine conditions, repeated correction was found necessary. To speed the diagnostic process we developed a method which allows one to rapidly track the machine optics. The method is based on measuring the propagation of $30 \mathrm{~Hz}$ modulated betatron oscillations downstream of a point of perturbation. Compared to the usual methods of dispersion or difference orbit measurement, synchronous detection of the beam displacement, as measured by beam position monitors, offers significantly improved speed and accuracy of the measurements. The beam optics of the accelerator was altered to decrease lattice sensitivity at critical points and to simplify control of the betatron function match. The calculation of the Courant-Snyder invariant from signals of each pair of nearby beam position monitors has allowed one to perform on-line measurement and correction of the lattice properties.

\section{Introduction}

The CEBAF accelerator $[1,2]$ is a 5 pass $\mathrm{CW}$ recirculator with beam power up to $800 \mathrm{~kW}$. It consists of a $45 \mathrm{MeV}$ injector, 2 superconducting (SC) linacs of $400 \mathrm{MeV}$ energy gain, and 9 arcs which connect the linacs for beam recirculation, so that the beam total energy is $4.045 \mathrm{GeV}$. After acceleration to the desired energy, the beam can be split and directed to 3 experimental halls for nuclear physics experiments.

During machine commissioning in early 1995 , it was found that the dispersion grew from pass to pass and the betatron function match from arc to arc was seriously degraded, causing a growth of the beam size and amplification of perturbations along the machine. Since the beam emittance is quite small, the transverse mismatch itself did not destroy the beam, but in higher passes the large. dispersion and betatron functions made operation overly sensitive to small energy and position fluctuations. To achieve stable operation, arc by arc dispersion correction was performed, and as machine conditions changed, repeated dispersion correction was found necessary. Typically, this was done with difference orbit diagnostics which enabled good control of the overall dispersion but left significant growth of betatron functions.

In light of the continuing need to correct the lattice functions, the beam position diagnostics were enhanced and an alternate tuning of the optics was
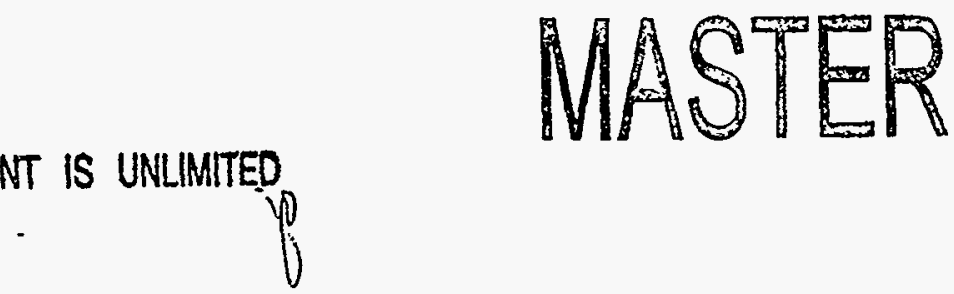


\section{DISCLAIMER}

This report was prepared as an account of work sponsored by an agency of the United States Government. Neither the United States Government nor any agency thereof, nor any of their employees, makes any warranty, express or implied, or assumes any legal liability or responsibility for the accuracy, completeness, or usefulness of any information, apparatus, product, or process disclosed, or represents that its use would not infringe privately owned rights. Reference herein to any specific commercial product, process, or service by trade name, trademark, manufacturer, or otherwise does not necessarily constitute or imply its endorsement, recommendation, or favoring by the United States Government or any agency thereof. The views and opinions of authors expressed herein do not necessarily state or reflect those of the United States Government or any agency thereof. 


\section{DISCLAIMER}

Portions of this document may be illegible in electronic image products. Images are produced from the best available original document. 
developed. To accelerate the measurements, we added an ability for the BPMs to perform phase detection of beam displacements excited by an external perturbation. This shortened the correction time by roughly an order of magnitude and allowed us to develop a method for performing on-line betatron match. As a result of the measurements and attempts to perform such corrections, we altered the design optics of the machine at locations where the focusing unavoidably changes during routine operation. Such alterations decreased the sensitivity of the optics to such changes, and we assigned quadrupoles responsible for global betatron match of the machine. An important diagnostic used for betatron match was based on the fact that Courant-Snyder invariant has to remain constant for the ideal optics and will change at all elements which have the wrong focusing strength. All this allowed us to correct the global properties of the machine optics and significantly improve beam quality. Now, it takes only about 8 hours to perform a total correction of the machine optics.

\section{Betatron mismatch and Courant-Snyder invariant}

One source of mismatch derives from the scale of the accelerator. During acceleration to the maximum energy the beam traverses a path length of more than 6 kilometers and is focused by almost 900 quadrupoles. Each quadrupole has a separate power supply which, on the one hand, creates many possibilities. for machine optics adjustment but, on the other hand, complicates the machine tuning and operation. For a machine of such scale, even if each small piece of the machine does not deviate very much from the optics design, combining many small errors together may produce something very different from the original intent.

Suppose a beam line exists with linear optics described by a design beta function $\beta(s)$. To understand the effect of a quadrupole error on the beam consider the change in the Courant-Snyder invariant,

$$
\epsilon=\beta(s) \theta^{2}(s)+2 \alpha(s) \theta(s) x(s)+\gamma(s) x^{2}(s),
$$

after the beam passes through a quadrupole with a focusing error, where $2 \alpha=$ $-d \beta / d s$ and $\gamma=\left(1+\alpha^{2}\right) / \beta$. To simplify formulas we will treat the quadrupole as a thin lens with focusing strength $\Phi=1 / F$ and error $\Delta \Phi$. Let $x$ and $\theta$ be the particle position and angle at entrance of the quadrupole for any orbit consistent with the linear optics. After passing through the quadrupole the particle angle will be perturbed from the nominal value by

$$
\delta \theta=-\Delta \Phi x .
$$

Substituting Eq. (2) into Eq. (1), we obtain

$$
\begin{gathered}
\epsilon^{\prime}=\beta(\theta+\delta \theta)^{2}+2 \alpha(\theta+\delta \theta) x+\gamma x^{2} \\
=\epsilon+2 \beta \theta \delta \theta+\beta \delta \theta^{2}+2 \alpha x \delta \theta=\epsilon\left(1+\beta \Delta \Phi \sin (2 \mu)+(\beta \Delta \Phi \cos \mu)^{2}\right),
\end{gathered}
$$


for the Courant-Snyder invariant of the perturbed orbit, where we took into account that the coordinate and angle of the unperturbed orbit can be expressed using $\epsilon$, and the betatron phase, $\mu$, by

$$
x=\sqrt{\beta \epsilon} \cos \mu, \text { and } \theta=-\sqrt{\frac{\epsilon}{\beta}}(\sin \mu+\alpha \cos \mu) .
$$

From Eq. (3) we finally obtain the following expression for relative change of the Courant-Snyder invariant

$$
\frac{\Delta \epsilon}{\epsilon} \equiv \frac{\epsilon^{\prime}-\epsilon}{\epsilon}=\beta \Delta \Phi \sin (2 \mu)+(\beta \Delta \Phi \cos \mu)^{2} .
$$

For a small quadrupole strength error the second term may be neglected and one observes, that the change of the invariant oscillates at double the betatron frequency.

Summing the effects of many quadrupoles, we can write from Eq. (3) that

$$
\begin{gathered}
\epsilon_{N}=\epsilon_{0} \prod_{n=1}^{N}\left(1+\beta_{n} \Delta \Phi_{n} \sin \left(2 \mu_{n}\right)+\left(\beta_{n} \Delta \Phi_{n} \cos \mu_{n}\right)^{2}\right) \\
=\epsilon_{0} \prod_{n=1}^{N}\left(1+\frac{1}{2}\left(\beta_{n} \Delta \Phi_{n}\right)^{2}+\sqrt{\left(\beta_{n} \Delta \Phi_{n}\right)^{2}+\left(\frac{\beta_{n} \Delta \Phi_{n}}{2}\right)^{4}} \sin \left(2 \mu_{n}+\psi_{n}\right)\right)
\end{gathered}
$$

where

$$
\tan \psi_{n}=\frac{\beta_{n} \Delta \Phi_{n}}{2} .
$$

To get an estimate of the betatron mismatch we will assume that the errors are independent for different quadrupoles and that the relative errors are scaled to the maximum quadrupole strength, $\Phi_{\max }$, equal for different quadrupoles. Then, supposing that the errors are sufficiently small, $N \overline{\left(\beta_{n} \Delta \bar{\Phi}_{n}\right)^{2}} \ll 1$, we can expand the product in Eq. (6) into a sum and after averaging we obtain an increase of the invariant after a passage of $N$ quadrupoles of .

$$
\frac{\overline{\Delta \epsilon}}{\epsilon} \approx \frac{1}{2} \sum_{n=1}^{N} \overline{\left(\beta_{n} \Delta \Phi_{n}\right)^{2}}=\frac{\overline{\Delta \Phi^{2}}}{\Phi_{\max }^{2}} \frac{1}{2 F_{\min }^{2}} \sum_{n=1}^{N} \beta_{n}^{2},
$$

and its relative variance,

$$
\frac{\sigma_{\epsilon}}{\epsilon}=\frac{\sqrt{\Delta \epsilon^{2}}-\overline{\Delta \epsilon}}{\epsilon} \approx \sqrt{\frac{1}{2} \sum_{n=1}^{N} \overline{\left(\beta_{n} \Delta \Phi_{n}\right)^{2}}}=\frac{\sqrt{\Delta \Phi^{2}}}{\Phi_{\max }} \sqrt{\frac{1}{2 F_{\min }^{2}} \sum_{n=1}^{N} \beta_{n}^{2}},
$$

Here $F_{\min }$ is the minimum focusing distance of the quadrupole $\left(F_{\min } \Phi_{\max }=1\right)$, and we neglected the second addend under the square root of Eq. (6) because $\beta_{n} \Delta \Phi_{n}<<1$. 
For the design specifications of the quadrupoles of $0.1 \%$, we obtain $\overline{\Delta \epsilon} / \epsilon \approx$ 0.02 and $\sigma_{\epsilon} / \epsilon \approx 0.2$ for one arc. Given the larger mismatch observed, the above analysis suggests some larger error source at several percent level in some arcs. It had been anticipated that there would be rematches at every pass by measuring the phase space using wire scanning techniques [3]. During the course of commissioning it was found difficult given installed diagnostics and small beam sizes to carry out these measurements rapidly and reproducibly. Difference orbit methods using RESOLVE code $[4,5]$ allowed adjustment to cure local problems, but were not sufficient to reduce the betatron mismatch after several passes through the machine.

\section{Alternate Optics}

In light of these difficulties, a new tuning of the optics was developed to reduce sensitivities to error sources and to isolate a few quadrupoles for adjustment to tune out the observed betatron growth. In parallel diagnostic tools were developed for rapid measurement of dispersion and betatron amplitudes so that this adjustment process could be carried out effectively and quickly.

From an optics point of view CEBAF [2] consists of 2 linacs (the so-called north linac, which is the first the beam traverses, and the south linac) and 9 arcs supporting recirculation (see Figure 1). Each linac is $240 \mathrm{~m}$ long and consists of 12.5 FODO cells. The recirculation arcs are separated vertically one above the other. Each arc consists of 4 periods performing a $180^{\circ}$ horizontal bend, and a spreader and a recombiner which perform a vertical separation of the beams with different energies, before and after the horizontal bend in the arc.

It was noticed during commissioning that the path length has long term variations (a few days or more). The magnitude of the path length change is about of 1-3 mm. Although this is a small relative change for six kilometers of beam transport, the accelerating phase for the final pass is changed by up to five degrees, yielding decreased beam quality. Nine so-called dog-legs, one for each recirculation arc, are used to correct path length. Each dog-leg consists of a 3 dipole chicane that creates a bump in trajectory to regulate path length within a limit of $10 \mathrm{~mm}$ for one dog-leg. The dipoles are magnets with parallel edges which do not have horizontal focusing. Nevertheless they produce vertical focusing which changes with the field strength of the magnets. Because' of the large vertical beta-function in the dog-legs in the original design, adjusting the doglegs significantly changes the vertical focusing throughout the entire machine. The largest effect is found in the first two arcs where the beta-functions may deviate from the design values by more than a factor of two for a change of one dogleg from its nominal field to its maximum field.

Another unstable region is the SC linacs. The accelerating gradients of many cavities are frequently adjusted taking into account the current state of the cavities, and a few cavities are always bypassed because they cannot operate properly. Thus, the profile of the accelerating gradient is always nonuniform and changes from day to day. Such changes are accounted for in the 
focusing of the first pass beam automatically, but the higher passes are also affected nontrivially. In addition, the fundamental mode couplers of the SC cavities exhibit quadrupole and skew-quadrupole components which change the beam focusing [6] as the accelerating gradient changes. In the first approximation the quadrupole components cancel for neighboring cavities, but the skew-quadrupole components are summed up through the entire linac and significantly affect the beam optics. In order to compensate for their effect, aircore skew-quadrupole magnets are installed throughout the linacs, on a common power supply for each linac.

For the design of the new optics, we changed the gradients of the quadrupoles in the spreaders and the recombiners only in ways such that the machine functions in the $180^{\circ}$ bends were not changed. The changes were concentrated in two main directions. First, we decreased the vertical beta-function inside the dog-legs (locatied in the spreaders) by about 3 times, which decreased their focusing effect. Second, we developed orthogonal knobs for optics correction, a . main feature of the alternate optics. Eighteen regions, one in each spreader and recombiner, allow one to do a smooth match throughout the entire machine. Each region has 4 quadrupoles assigned for correction of the beta-function and its derivative, for both horizontal and vertical planes. To decouple the horizontal and vertical corrections there is a large difference in the horizontal and vertical beta-functions inside these quadrupoles. Taking into account that the beam envelope oscillates at twice the betatron frequency, the 2 quadrupoles of each correction set, horizontal or vertical, are shifted by $45^{\circ}+n 90^{\circ}$, yielding orthogonality of their effects and, correspondingly, requiring minimal changes of their gradients to perform a betatron match. Figure 2 demonstrates behavior of beta-function and dispersion for the spreader of ARC1.

Also, the requirement of unit transfer matrix for an entire arc and the requirement of having special phase advances for the spreaders or recombiners were relaxed. Separate treatment of the linacs was thereby permitted, allowing us to decrease beta-functions there. Figure 3 shows the change of beta-functions for the fifth pass in both linacs. The gain for the south linac is larger. One can see that the maximum beta-function has been decreased more than 2 times for the south linac. This decrease of the beta-function decreases both the sensitivity of the optics to changes of the accelerating gradient profile and to the effect of the coupling due to skew-quadrupole fields of the SC cavities.

\section{Instrumentation for Performing Dispersion and Betatron Match}

Our fast diagnostic system provides real-time measurements of many quantities of interest in commissioning the accelerator. Examples are: measurement and correction of the dispersion and the betatron transfer matrices, detection of locations of beam scraping, and measurement and correction of horizontalvertical coupling. In the future it should be possible to measure individual cavity phases using the same general idea, with small perturbations to normal beam delivery, yielding the minimum possible beam energy spread. 
Performing measurements on the accelerator optics practically always implies some kind of differential measurements: for measurements of dispersion one needs to measure how the orbit is changed as result of a beam energy change; for measurements of the beam transfer functions one needs to measure how the orbit will be changed after the beam receives a transverse kick. Thus, the general idea of the measurements is to modulate a specific parameter and to measure a response to this signal on downstream BPMs. Beam position monitors are the most frequent diagnostic at the CEBAF accelerator, allowing one to perform detailed measurements throughout the entire machine.

The modulation frequency is chosen based on the following considerations. First, the modulation frequency has to be a subharmonic of the power line frequency to suppress its electromagnetic interference. Second, it should be at the highest possible frequency to decrease the deleterious effects of slow beam drifts. Because line-synched $60 \mathrm{~Hz}$ pulsed beam has been the main mode of beam operations during CEBAF commissioning; we chose a modulation frequency of $30 \mathrm{~Hz}$, at the first subharmonic of the power line frequency. As can be seen below, such a choice simplifies calculation of the BPM response to the modulation.

A schematic of the system is shown on Figure 4. The $30 \mathrm{~Hz}$ modulation is generated by a function generator that resides in the machine control center and is phase locked to the beam pulses. The modulation is delivered uniquely to one of the modulation devices by computer controlled switches, depending on the type of measurement to be accomplished. Presently, there are two types of modulation used: beam energy modulation performed by changing the accelerating gradient of the superconducting cavities and transverse beam modulation performed by air-core dipole correctors.

The final four cavities of the injector linac and the final eight cavities of the first CEBAF linac are used for the energy modulation. Energy modulation is achieved by switching the modulating voltage to an analog input into the gradient feedback loop of the RF control modules. The modulation voltage yields an initial relative energy change of about $2 \times 10^{-3}$, which depends somewhat on the pass number and arc location. It should be noted that the relative energy modulation remains constant after each passage through the first linac.

There are three groups of the correctors located (1) at the beginning of the injector at an energy of $5 \mathrm{MeV}$ ( 1 vertical and 1 horizontal corrector), (2) at the end of the injector at an energy of $45 \mathrm{MeV}$ (2 vertical and 2 horizontal correctors), and (3) at the beginning of ARC1 at energy of $445 \mathrm{MeV}$ (2 vertical and 2 horizontal correctors). Each set of correctors is located at a place with approximately 10 times larger energy, allowing one to perform accurate measurements throughout the entire machine in spite of adiabatic damping due to acceleration, which decreases the amplitude of betatron motion inversely proportional to the square root of energy change. Usually we excite beam motion with an amplitude of about $3 \mathrm{~mm}$, which after acceleration by 10 times will die down to about $1 \mathrm{~mm}$. The third set of correctors, located after the first pass 
of the north linac after the vertical beam spreading, is mostly frequently used. This set performs almost all measurements dealing with betatron match of the arcs and the subsequent passes through the north and south linacs.

The beam position measurements are made with monitors that detect the fundamental beam frequency on four wires $(X+, X-, Y+$, and $Y-)$, sampling the voltage induced on each wire and then converting the sampled voltages to $X$ and $Y$ positions relative to the center of the beam pipe and to the beam current. Two types of electronics with associated software are used at CEBAF to perform the data acquisition and position calculation: 4-channel electronics [7] and switched electrode electronics (SEE) [8]. Currently, the measurements are implemented only for the 4-channel electronics. Most of these BPMs are located in the arcs, with a few in other portions of the accelerator. The SEEs are concentrated in the linac sections of the accelerator; processing the modulation * signals for these BPMs will be implemented soon. All told there are approximately $450 \mathrm{BPMs}$ distributed across a network of 20 single-board processors called input-output controllers (IOCs) in a VME-based system using VxWorks and EPICS as the control software [9]. The 4-channel BPMs interface to the VME system via CAMAC.

Data for the $30 \mathrm{~Hz}$ measurements are acquired at a $60 \mathrm{~Hz}$ rate and stored in a buffer for processing. The 4-channel BPMs in the arcs are located on 5 separate beam pipes and in order to reduce costs, $5 \mathrm{BPMs}$ (one from each pass) share a single set of electronics. A multiplexer is used to select the BPM for the data acquisition process. In order to provide $1 \mathrm{~Hz}$ position update rates to the operators, the number of points acquired for each BPM in the arc region is limited to 12 , which is the usual choice for the differential beam position measurements. For BPMs in some of the other regions of the accelerator where multiplexing is not used, the number of $60 \mathrm{~Hz}$ data samples acquired per second is 24 .

To find an amplitude of the $30 \mathrm{~Hz}$ modulation, an IOC computes a difference between each two sequential measurements for each wire so that the differential signal is equal to

$$
u_{d}=\frac{(-1)^{s}}{N} \sum_{n=1}^{N}(-1)^{n} u_{n}
$$

where $N$ must be an even number (usually 12), $s$ is 0 or 1 depending on the sign given by the synchronization system, and the $u_{n}$ are the signals measured from a wire. Using these differential signals and the mean signals from each wire the IOCs calculate both the general beam position, which should not be changed by $30 \mathrm{~Hz}$ modulation, and the differential beam position displaying the amplitude of $30 \mathrm{~Hz}$ motion.

Because the modulating voltage is locked to the beam pulses, the ADC -measurements of the BPM signals are automatically synchronized to the modulation. The synchronization of readings for different IOCs is accomplished by broadcasting a "sign bit" of the $30 \mathrm{~Hz}$ modulation, $s$, which allows one to obtain 
the sign of the differential response. Consistent with the load on the network, one of the IOCs, called the master IOC, measures the sign of the $30 \mathrm{~Hz}$ signal and sends synchronization messages (see Figure 4) every ten seconds. The messages are sent to the other asynchronously running slave IOCs with a check that either the message arrives in time, or it fails to arrive in time. In the event that the message fails to arrive, the IOC maintains synchronization with its local clock. As the local clocks drift at a rate that up to fourteen broadcasts can be lost before the desynchronization occurs, synchronization of the modulation is assured; failure in the arrival of individual messages is a reasonably low probability event.

The displays for the difference orbits are straightforward replications of the normal orbit displays at CEBAF. Just as the general BPM screens show beam positions and beam current, the differential screens show differential beam positions and differential beam current. Normally, the differential beam current is equal to zero (because the modulation does not change the beam current), but when the beam is scraped, the beam current depends on beam position and the differential beam current shows where scraping starts. Just as for general BPM displays, there are displays for relative data showing the change of differential positions compared to a previously saved differential orbit. This is useful for comparing the current machine state with a previous one. Therefore, analogously to the case of having stored good orbits through the accelerator from the usual BPM outputs, it is possible to save the proper difference orbits throughout the machine, and provide tuning information much like the usual transverse tune in a ring accelerator. Such difference differencing can be rapidly used to locate off normal quadrupole settings, a facility that should be especially useful as energy changes are made in the accelerator.

\section{Performing Dispersion and Betatron Correction}

Performing the dispersion correction was greatiy simplified after this system was completed. In order to explain the correction procedure, Figure 5 shows the calculated horizontal and vertical dispersions and beta-functions throughout ARC1 and the downstream linac. The lattices of all other arcs are built on the same principle. To perform the dispersion correction one needs to excite $30 \mathrm{~Hz}$ modulation of beam energy and, consequently, the horizontal and vertical differential BPM beam positions will be proportional to the horizontal and vertical dispersions. As one can see from Figure 5 the vertical dispersion is different from zero in the spreaders and recombiners alone. Thus to correct the vertical dispersion an operator needs to adjust the currents in the two quadrupoles of each spreader and recombiner responsible for the vertical dispersion match. Each quadrupole excites different dispersion patterns in downstream BPMs and it is comparatively easy to zero dispersion after a few iterations through these two quadrupoles. Such corrections are significantly simplified due to the fact that the BPM screen responds practically instantly. Analogously, for the horizontal 
dispersion correction one needs to adjust the two quadrupoles responsible for horizontal dispersion correction. Taking into account that the dispersion errors propagate throughout the entire machine, the corrections must begin in ARC1 and proceed downstream arc-by-arc. It is important to do a good match on each step but it is even more important that, due to global measurements of the dispersion, the errors of previous steps (due to finite BPM resolution) are compensated on following steps, preventing the accumulation of errors. Note that corrections of the horizontal and vertical dispersions are decoupled but they affect the betatron function match, which should be performed after completing the dispersion match.

Performing the betatron match is more complicated. It is based on the fact that the Courant-Snyder invariant must remain constant for the ideal lattice. The general idea is to excite the betatron motion and observe how the invariant changes during beam transport. Changes will occur at elements which have a focusing different from the design model. As can be seen from Eq. (5), the perturbation depends both on the focusing error and the betatron phase. Thus measurements with different betatron phases must be performed to obtain complete information on the betatron match.

In addition to the differential BPM screens, we built a program which calculates an effective betatron motion amplitude from the differential measurements of two sequential BPMs and plots this information for the entire machine on one screen. This amplitude is proportional to the square root of the CourantSnyder invariant and has an additional beam energy normalization so that the amplitude remains constant with beam acceleration. For calculations of the normalized amplitude of horizontal betatron motion at the $i$-th BPM, we use the following equation

$a_{X_{i}}=\sqrt{\frac{\beta_{0} E_{i}}{E_{0}}\left[\beta_{x_{i}}\left(\frac{x_{i+1}-M_{11}^{i, i+1} x_{i}}{M_{12}^{i, i+1}}\right)^{2}+2 \alpha_{x_{i}}\left(\frac{x_{i+1}-M_{11}^{i, i+1} x_{i}}{M_{12}^{i, i+1}}\right) x_{i}+\frac{1+\alpha_{x_{i}}^{2}}{\beta_{x_{i}}} x_{i}^{2}\right]}$,

where $M_{k l}^{i, i+1}$ are the transfer matrix elements between BPMs $i$ and $i+1$ for the design lattice $(k, l=1,2 . .6), \beta_{x_{i}}$ and $\alpha_{x_{i}}$ are the beta-function and its negative derivative halved for the design lattice at the position of $i$-th BPM, $E_{i}$ and $E_{0}$ are the current and initial energies of the beam, and $\beta_{0}=10 \mathrm{~m}$ is a convenient normalization. Analogous formula are used for calculations of the normalized amplitude of the vertical motion. The program cycles through both horizontal (or vertical) correctors and plots four curves for the normalized amplitudes of the horizontal motion. Two of these curves are produced by measurements obtained from excitation of correctors 1 and 2 , and the other two are built from linear combination of the signals $x_{1}+x_{2}$ and $x_{1}-x_{2}$. Taking into account that correctors 1 and 2 are shifted by about $90^{\circ}$ in betatron phase allows one to obtain measurements for four betatron phases shifted by $45^{\circ}$ in sequence by performing only measurements for two of them. This accelerates the mea- 
surements by a factor of two because the time of the calculations is negligible compared to the duration of the measurements. The time duration of the measurements is determined by EPICS software and is about $0.4 \mathrm{~ms}$ per channel, which for $X$ and $Y$ positions of 1000 BPM readings implies a measurement time for one curve of about $0.4 \mathrm{sec}$, only slightly below the $1 \mathrm{sec}$ sampling time of the BPM measurements. It follows from Eq. (5) that for the chosen betatron phase advances between the curves, if we have a error in focusing at least one of the curves will go down and at least one will go up. We assumed here that the errors are sufficiently small so that the second addend in Eq. (5) can be neglected. The purpose of the correction is to adjust quadrupoles responsible for betatron match so that each of four normalized amplitudes would have a constant level throughout the entire machine.

As in the case of dispersion correction, the correction of betatron functions is performed arc-by-arc. To match beta-functions of two sequential regions of the machine an operator must adjust two corresponding quadrupoles assigned for betatron match between these regions so that all four curves of normalized amplitude are on the same level for these two regions. When an operator proceeds to the next region he must align the curves to the first region to avoid accumulated errors. Corrections for horizontal and vertical betatron matches are highly decoupled so that it requires no more than two iterations to perform a match for both planes. Note that this procedure does not correct errors inside a region. It is used to correct the betatron functions globally so that betatron functions for the entire machine remains close to the design. This procedure does not correct phase advances of the betatron motion, it corrects only the beam envelopes which, from a practical point of view, are much more important for the linear accelerator.

Figure 6 shows normalized amplitudes for horizontal and vertical motion for a horizontal perturbation after correction of the betatron match. One observes that the normalized amplitude does not changed significantly in one arc and errors are not accumulated along the machine. The normalized amplitude is usually amplified by a factor of three to ten times without the benefit of the matching. Note that there are occasional large spikes on the curves which are associated with BPMs that are not functioning properly.

Usually an operator watches screens for horizontal and vertical motion'simultaneously and can see an effect of the horizontal-vertical coupling in the process of correcting the betatron function, and horizontal-vertical coupling is adjusted during and at the end of the the betatron match. An example of the. effect appears in Figure 6 where the amplitude of the vertical motion is slowly growing along the machine for an initial horizontal perturbation. Although one can still see a residual coupling, its size is significantly reduced compared with the initial state of the optics.

\section{Discussion}

Although during dispersion and betatron function matching we did not mon- 
itor what happened to the beam sizes, performing the match resulted in a small beam size throughout the machine. There is practically no emittance growth during beam transport. Figure 7 shows the results of beam size measurements along the machine and their predictions based on the measurements of the emittance in the injector. Measurements were done by scanning a $50 \mu \mathrm{m}$ wire throughout the beam with $50 \mu \mathrm{m}$ steps. One can see that there is a good agreement between measurements and predictions if one takes into account the final accuracy of the profile measurements due to the resolution of the profile monitor of about $30-50 \mu \mathrm{m}$.

The main result of this work is that we developed instrumentation and procedures which allow one to carry out a test of the beam optics quickly, and if necessary to correct the optics rapidly.

We would like to thank A. Hutton, J. Bisognano, C. Sinclair, M. Tiefenback and D. Douglas for fruitful discussions and support of this work. We are grateful to K. Crawford, R. Legg, W. Merz, and M. Lehmann for help with installing and tuning the hardware. We greatly appreciate the help of the whole operations department, which helped us by their continuous efforts. *This work was supported by U.S. D.O.E. contract \#DE-AC05-84ER40150.

\section{References}

[1] Douglas, D. R., R. C. York, and J. Kewisch, CEBAF-PR-89-008; Proc. of the 1989 Particle Accelerator Conference, pgs. 557-559 (1989)

[2] Bowling, B., D. R. Douglas, L. H. Harwood, J. Kewisch, D. V. Neuffer, J. Y. Tang, and R. C. York, Proc. of the 1991 Particle Accelerator Conference, pgs. 446-448 (1991)

[3] Douglas, D. R., and R. Legg, CEBAF Internal Technical Note 96-024 (1996)

[4] Lee, M., "RESOLVE User Guide"

[5] Yunn, B., R. Li, and S. Simrock, Proc. of the 1995 Particle Accelerator Conference and Intl. Conf. on High-Energy Accelerators pgs. 2297-2299 (1995)

[6] Li, Zenghai, Proc. of the 1993 Particle Accelerator Conference, pgs. 179-181 (1993)

[7] Hofler, A. S., et. al., Proc. of the 1999 Particle Accelerator Conference, pgs. 2298-2300 (1993)

[8] Powers, T., et. al., Proc. of the 1996 Beam Instrumentation Workshop, to be published 
[9] Dalesio, L. R., et. al., Proc. of the Intl. Conf. on Accel. and Large Exptl. Physics Control Systems, pgs. 278-282 (1992)

\section{Figure captions}

Figure 1. Layout of the CEBAF accelerator.

Figure 2. Beta-functions and dispersion for the spreader of ARC1. Locations of quadrupoles assigned for the betatron match are shown by arrows.

Figure 3. Comparison of betatron functions for last pass in the north (left) and south (right) linacs for the design optics (top) and the alternate optics (bottom).

- Figure 4. Schematic of the $30 \mathrm{~Hz}$ measurement system

Figure 5. Dispersion and betatron functions in ARC1 and first pass of the south linac

Figure 6. Courant-Snyder invariant after correction

Figure 7. Normalized emittance plotted as function of the profile monitor number. Each arc has two profile monitors so that eighteen results are shown for the nine arcs in the order the beam encounters them. The dotted lines show the emittance measured in the injector. $x$-horizontal emittance, + vertical emittance 


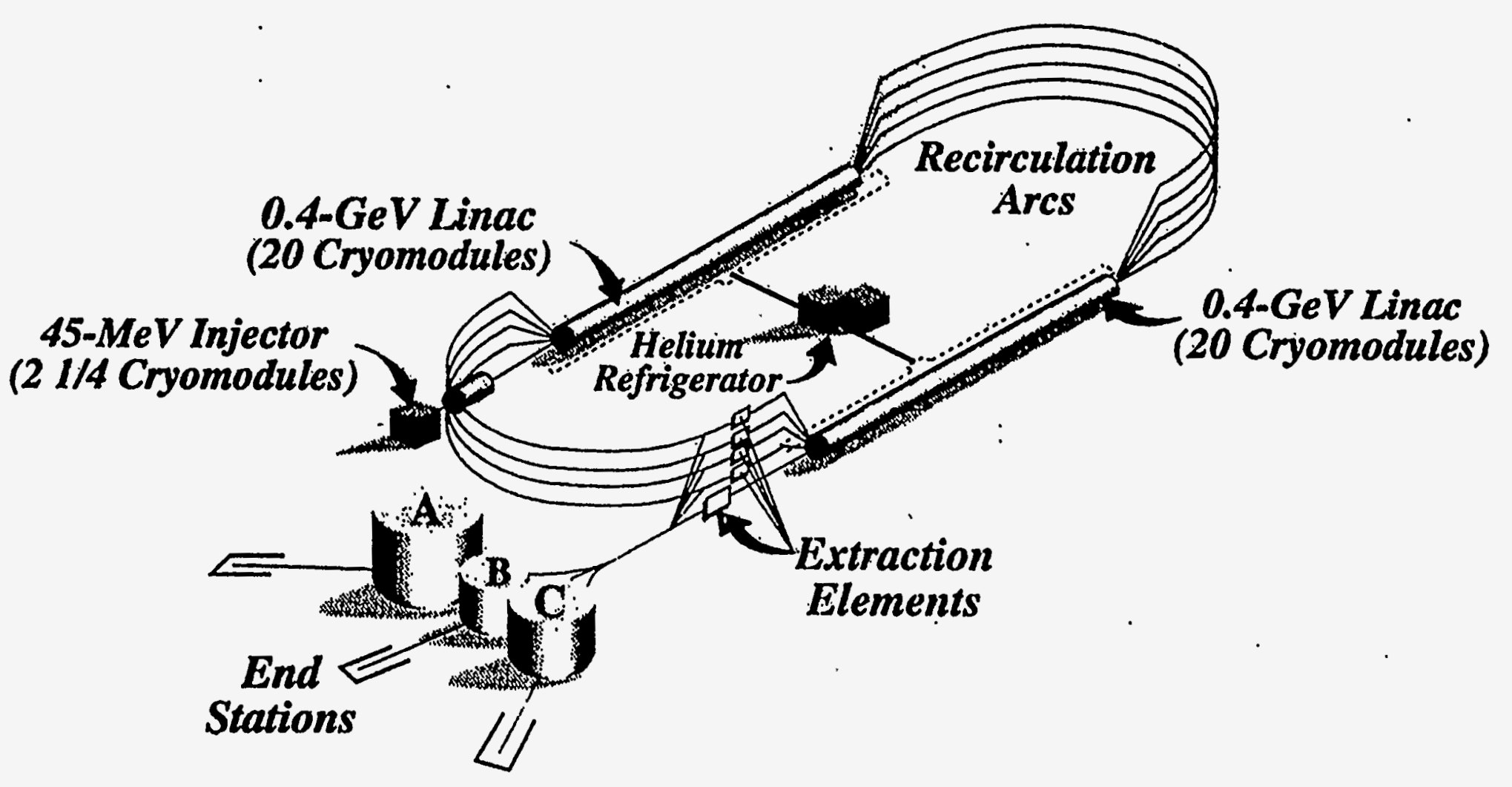



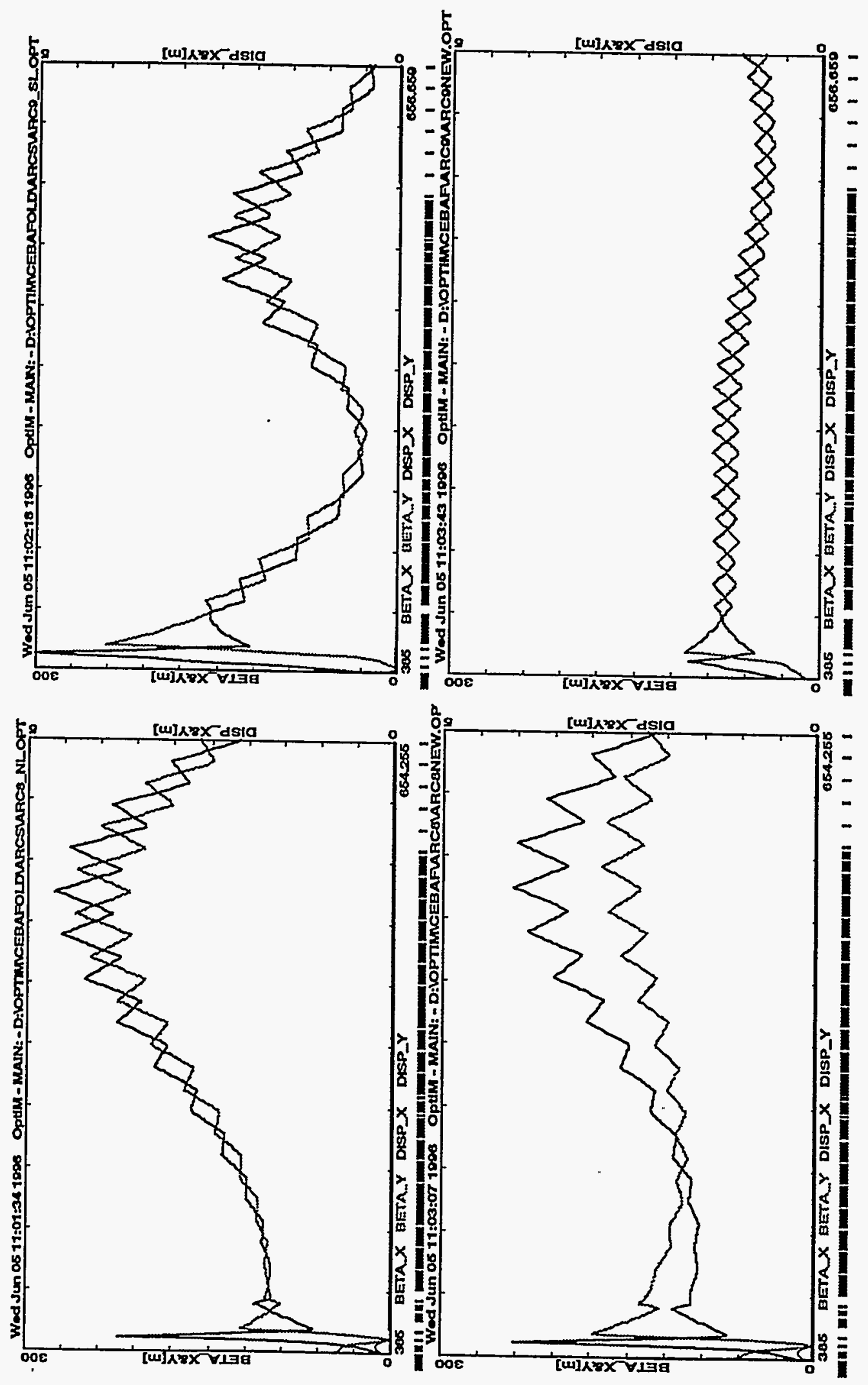

峁 


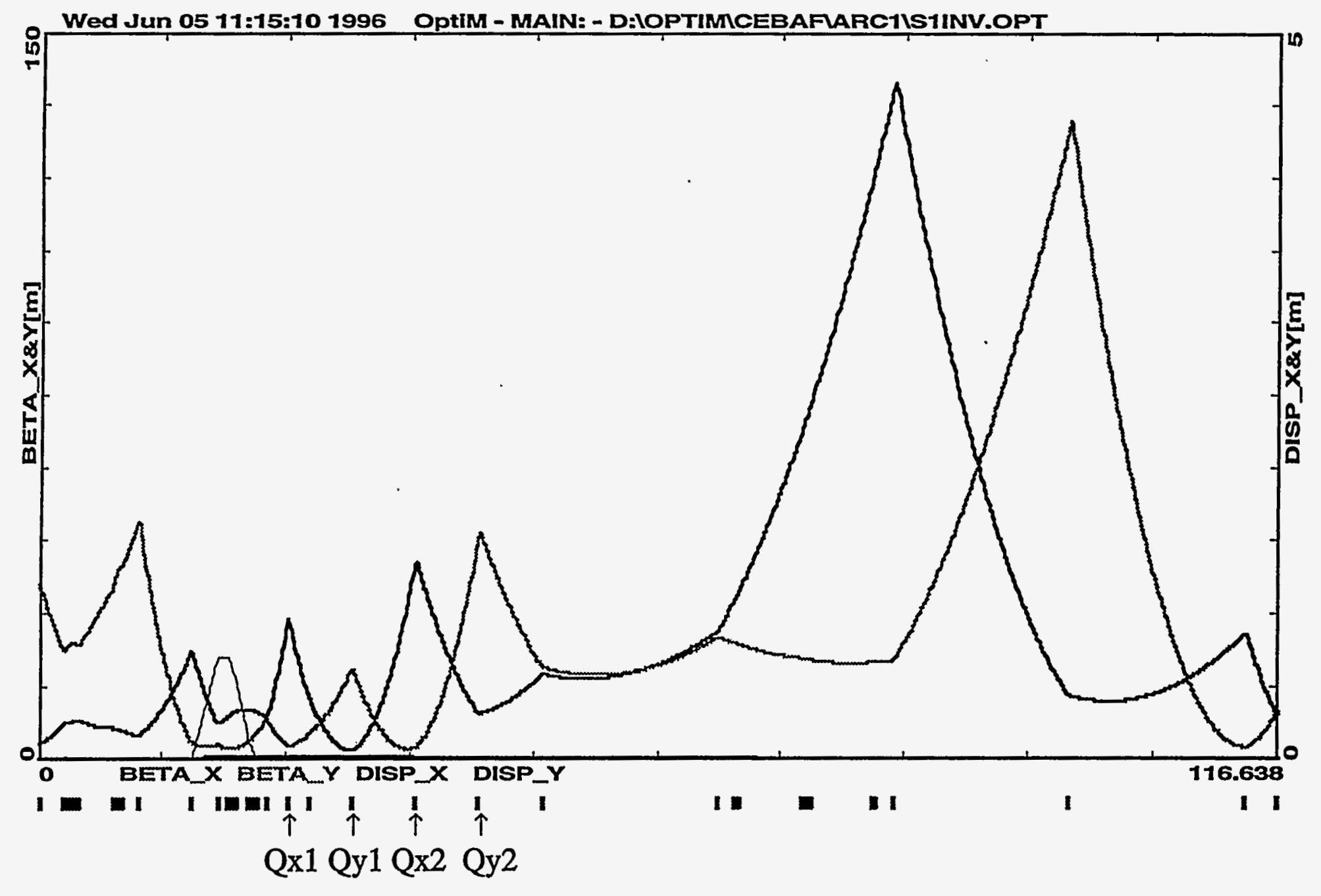

Figure 3. 


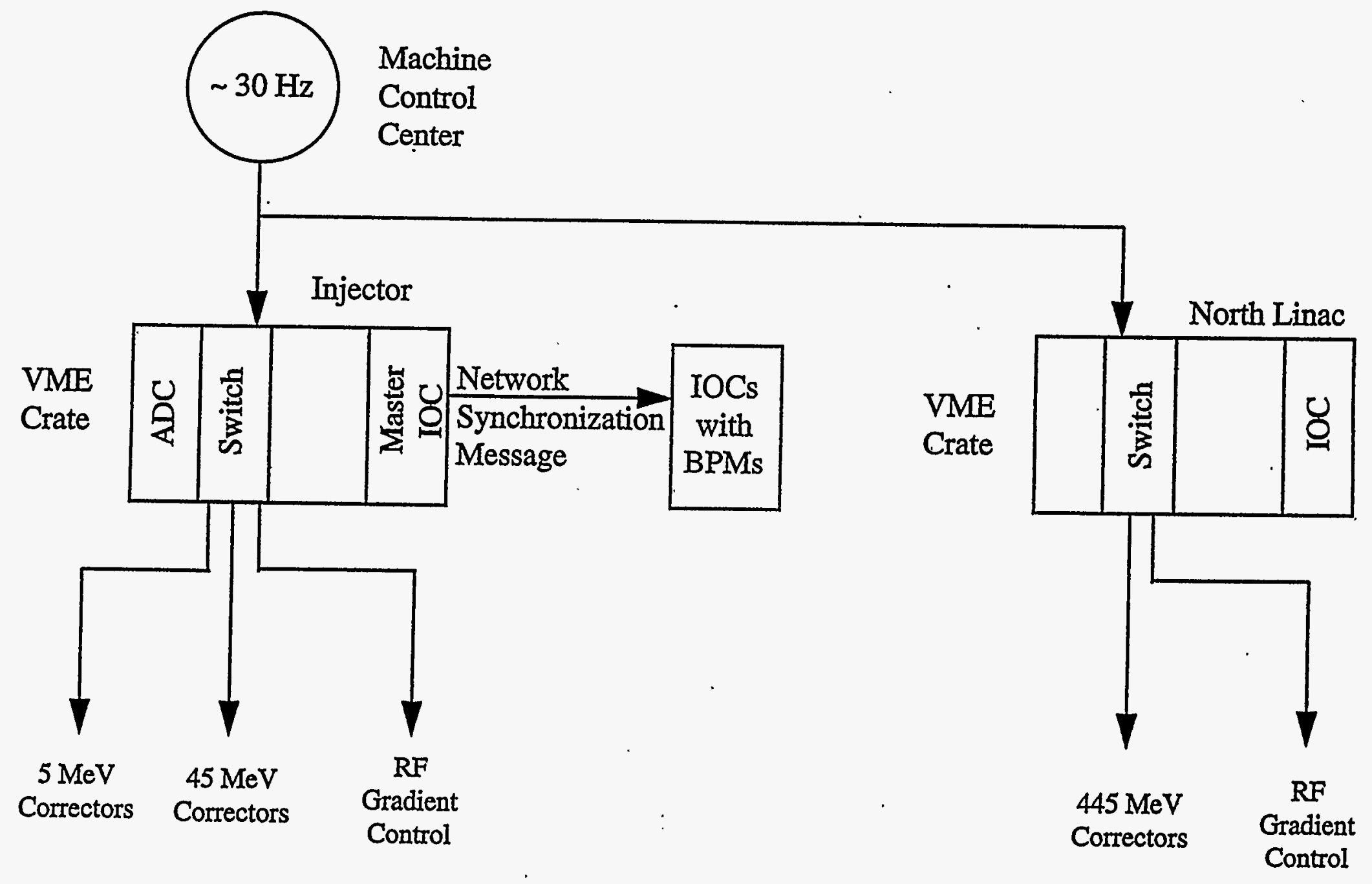




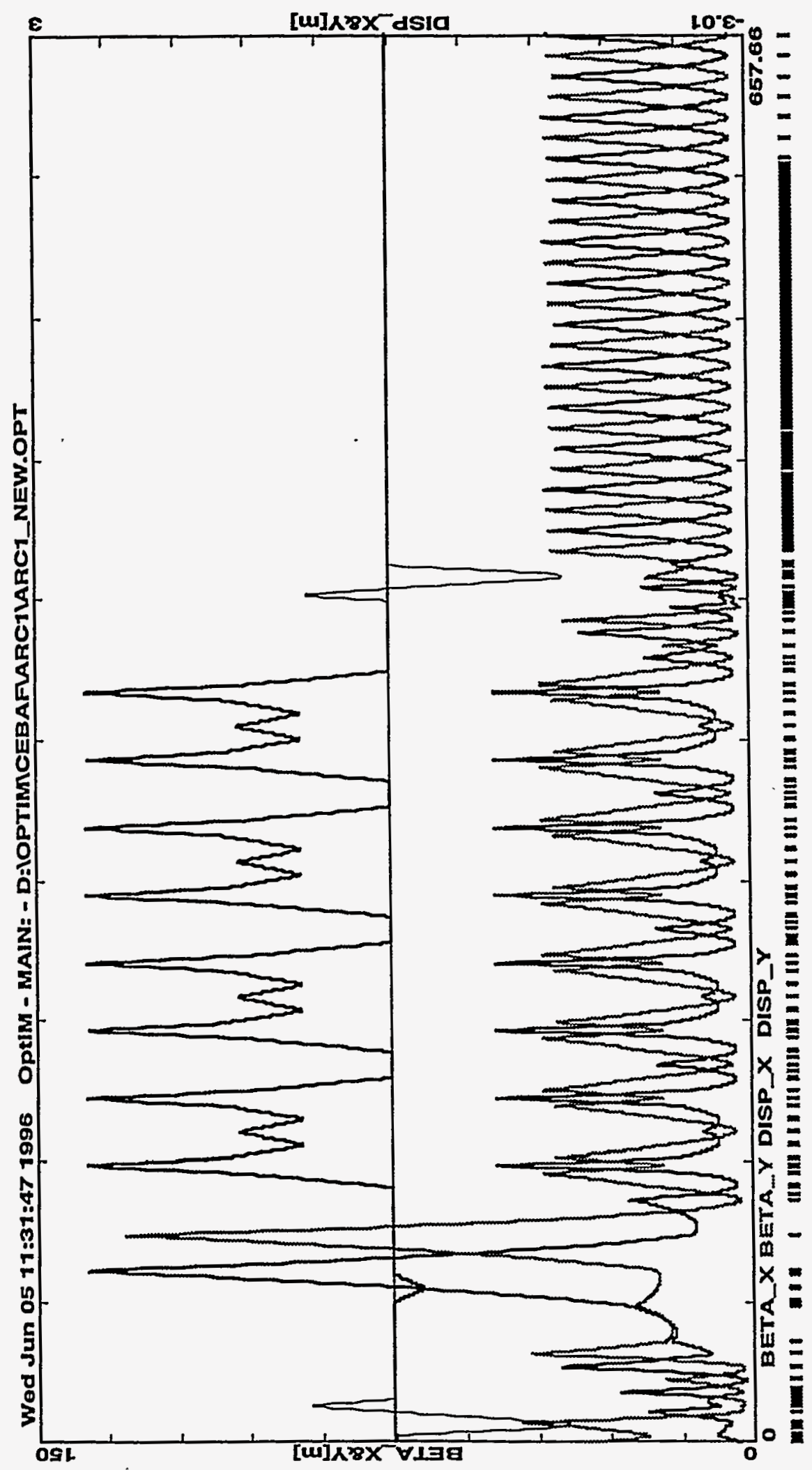



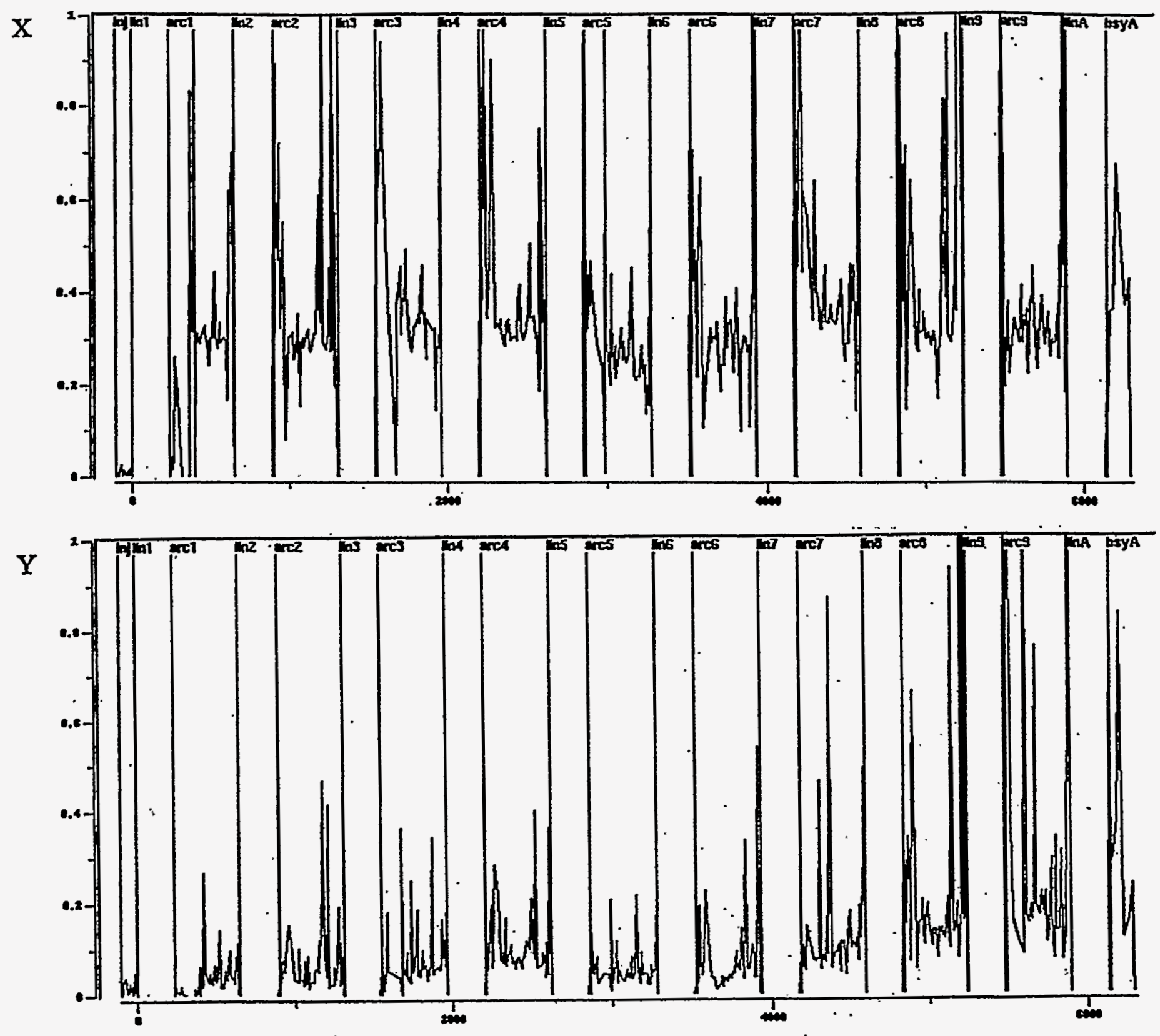

Fig.6. 


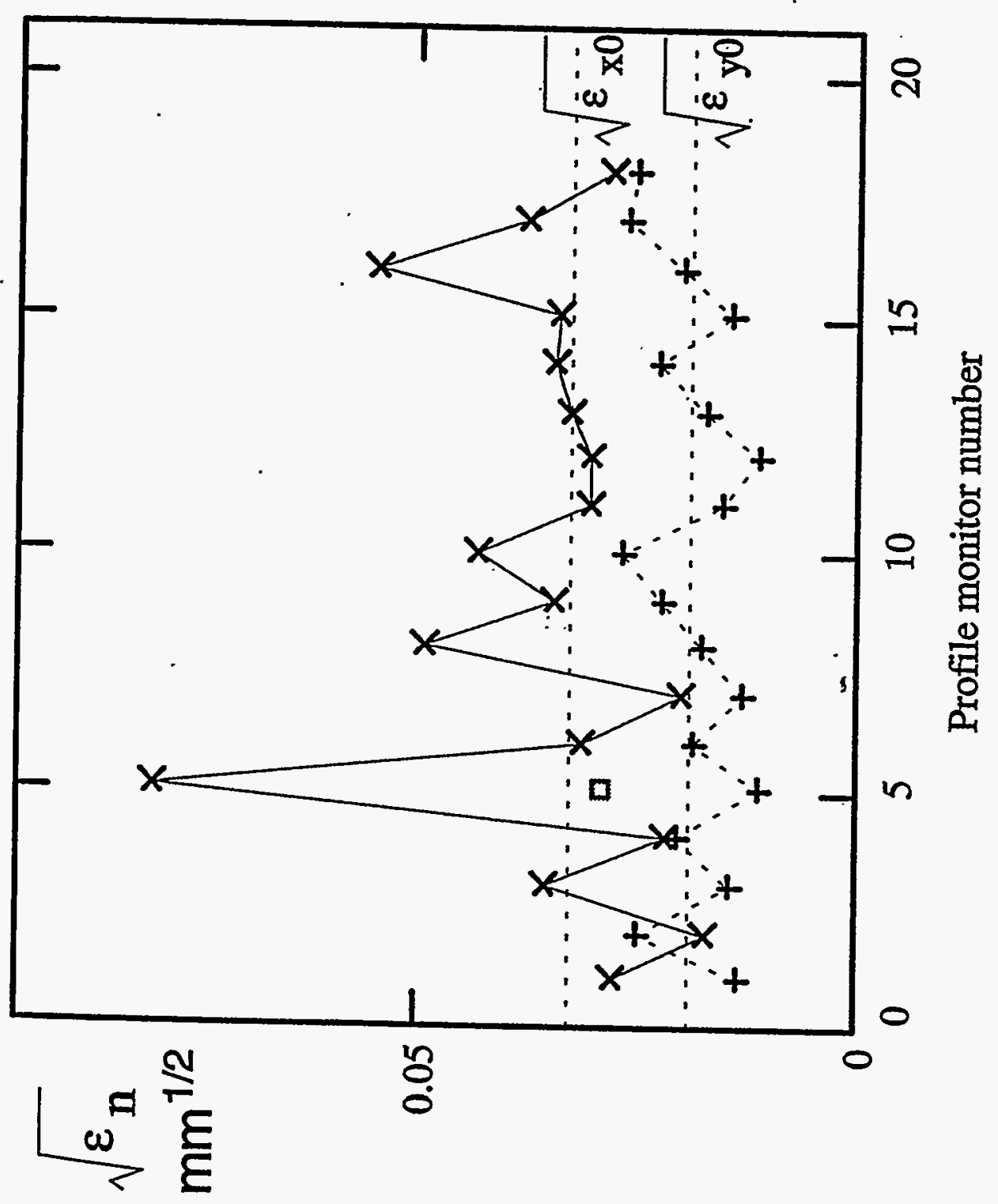




\section{RECEIVED \\ OCT 031996 \\ OSTI}

This report has been reproduced from the best available copy.

Available to DOE and DOE contractors from the Office of Scientific and Technical Information, P.O. Box 62, Oak Ridge, TN 37831; prices available from (615)578-8401, FTS 626-8401.

Available to the public from the National Technical Information Service, U.S. Department of Commerce, 5285 Port Royal Rd., Springfield, VA 22161.

Price: Printed Copy AO2

Microfiche A01

$\therefore$ 